\title{
Sub-mW Reconfigurable Interface IC for Electrochemical Sensing
}

\author{
Sara Ghoreishizadeh, Cristina Boero, Antonio Pullini, Camilla Baj-Rossi, Sandro Carrara, and Giovanni De Micheli
}

\begin{abstract}
The IronIC project has the aim of developing a fully implantable and remotely powered platform for the realtime monitoring of human metabolites. In this paper we present a mixed-signal interface IC for the electrochemical sensing data acquisition chain. The IC controls and reads out up to five biomolecular sensors, by receiving commands from a standard interface to conduct chronoamperometry $(\mathrm{CA})$ and cyclic voltammetry $(\mathrm{CV})$. Different voltage profiles are generated by using a single fully on-chip reconfigurable waveform generator, while the measured data are digitized. The IC is realized in $0.18 \mu \mathrm{m}$ CMOS technology. Electrical measurements show that the linear readout current range is $\pm 1650 \mathrm{nA}$ with 8-bit resolution. The cyclic voltammetry of potassium ferricyanide and the chronoamperometry of hydrogen peroxide have been successfully performed with the interface. The IC consumes 0.92 $\mathrm{mW}$ from $1.8 \mathrm{~V}$ supply voltage, making it suitable for remotely powered and implantable applications.

Index Terms-Interface IC, Electrochemical sensing Control and Readout, Cyclic Voltammetry, Chronoamperometry.
\end{abstract}

\section{INTRODUCTION}

Fully implantable devices for the monitoring of human metabolism require high level of miniaturization and integration. The electrochemical detection of the target molecules can definitely solve the issue of small and minimally-invasive implants. The IronIC project has the aim of developing a fully implantable and remotely powered platform for the real-time monitoring of human metabolites. The target molecules are principally but not limited to glucose, lactate, ATP, arachidonic acid, and bilirubin. The project is quite ambitious and presents several challenges:

- the continuous monitoring of multiple metabolites

- the integration of heterogeneous systems and technologies on the same platform

- the biocompatibility

- the remote powering through the skin.

The final system consists of three devices: a fully implantable sensors array for the metabolite detection, a wearable patch for the remote powering and data acquisition, and an interface dedicated to display the measurements on tablets and smartphones. A conceptual sketch is depicted in Fig. 1.

The authors are with the Integrated System Laboratory of the EPFL, Lausanne, Switzerland. (Email: seyedehsara.ghoreishizadeh@epfl.ch).

The research has been financed by the project IronIC++ with a grant from the Swiss Nano-Tera.ch initiative and evaluated by the Swiss National Science Foundation, and from the SNF Sinergia project (CRS 112-147694/1).

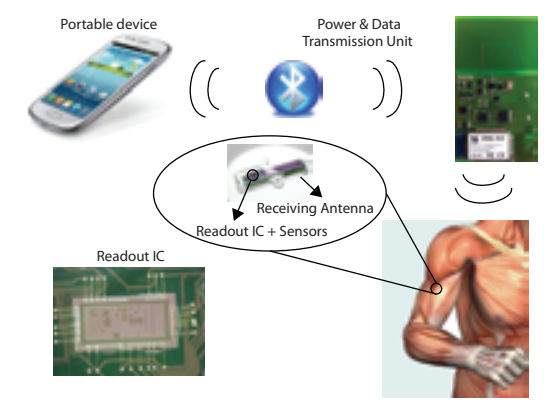

Fig. 1. Conceptual sketch of the final system for the IronIC project.

Based on the fact that the chemical detection is transduced in an electrical signal, the implantable platform requires a control unit and a readout electronics to perform the detection. The multi-target detection needs the integration of different detection methods within the readout IC. Chronoamperometry (CA) and cyclic voltammetry $(\mathrm{CV})$ are the two most common electrochemical techniques used to measure the concentration of endogenous (i.e. glucose, lactate) and exogeneous (i.e. drugs) molecules in the human body [1-3].

Low power interface ICs for glucose and neurotransmitters detection through CA were presented in [4-7]. Interface ICs for $\mathrm{CV}$, instead, include only the sensor readout, while using external waveform generators for the sensor control [8-12] . Li et al. reported an interface IC including all CA and CV control and readout parts. However it consumes about $20 \mathrm{~mW}$, which make it not suitable for remote powering and implantable applications [13]. A fully-integrated and low-power readout IC to perform both $\mathrm{CA}$ and $\mathrm{CV}$ control and readout has not been introduced in literature, to the best of our knowledge.

In this paper we present a new reconfigurable sub-mW interface IC, controlling and reading out five bimolecular sensors in both $\mathrm{CA}$ and $\mathrm{CV}$ techniques with a fully digital output. The interface IC controls also a $\mathrm{pH}$ and a temperature for sensor calibration. It works with a $1.8 \mathrm{~V}$ supply voltage and has a single readout circuit for both $\mathrm{CV}$ and $\mathrm{CA}$. The reconfigurable sensor control part is implemented fully onchip without any need of external clock or bias generator. The measured signal of all the electrochemical sensors is digitized through a fully-on chip sigma-delta analog to digital converter (ADC). The IC configures the sensor array and performs $\mathrm{CV}$ or CA based on the received commands, and streams out the measured current. To validate the interface IC, $\mathrm{CV}$ and $\mathrm{CA}$ 


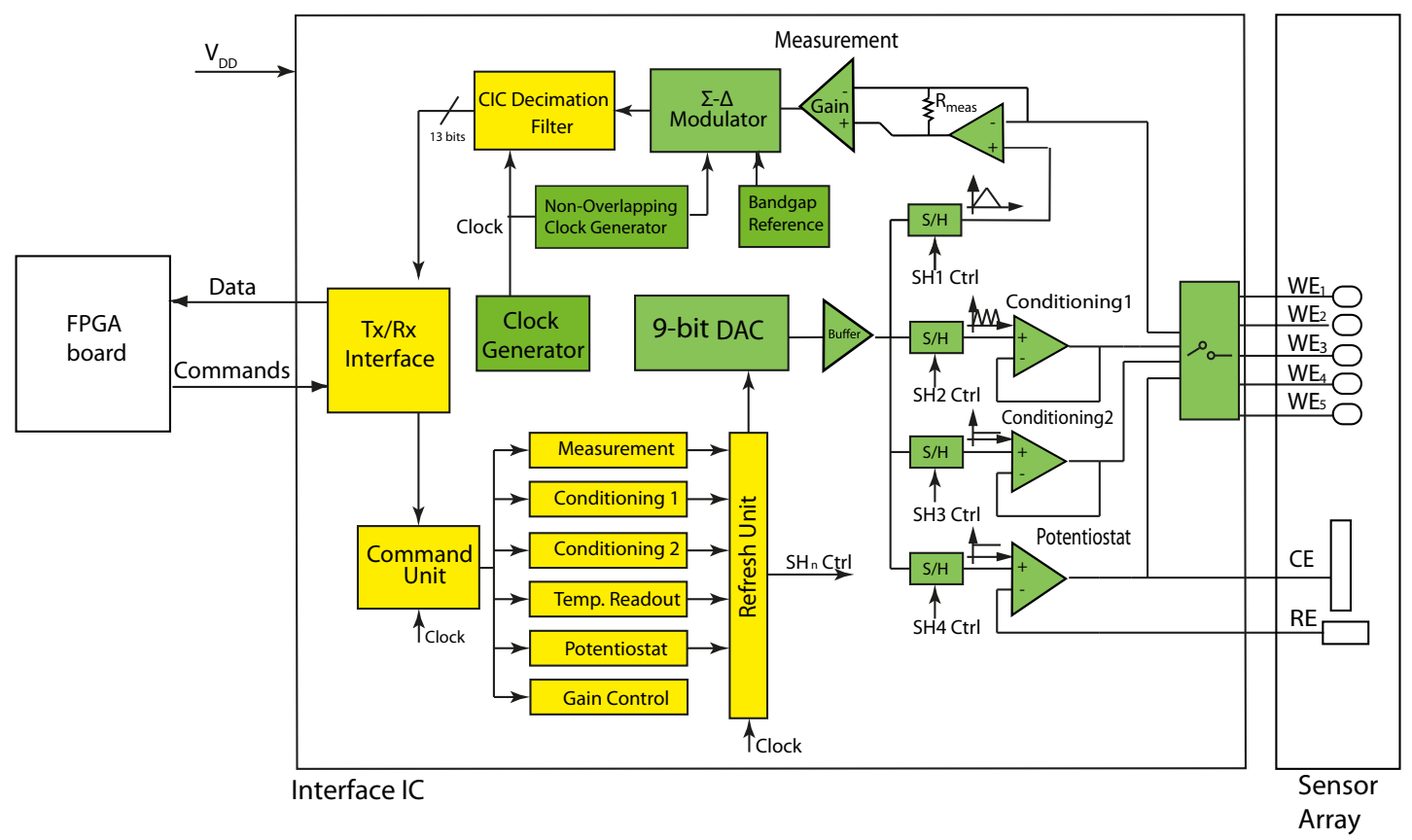

Fig. 2. The schematic view of the interface IC. The blocks in green and yellow are implemented in full-custom analog and semi-custom digital, respectively.

measurements are performed on the electrochemical sensors by using potassium ferricyanide and hydrogen peroxide as models of target molecules.

The design of the interface IC is presented in the following section. The electrical measurement are presented in Section III. Both CA and CV measurements are reported in Section IV. The summary and conclusions are given in Section V.

\section{INTERFACE IC}

The block diagram of the mixed-mode interface IC is shown in Fig. 2, where the analog and the digital blocks are shown in green and yellow, respectively. The IC is in contact with five bio-molecular sensors. The interface IC is configured for a measurement according to the Commands, and it streams the results out on the Data pin. In this section we explain three main parts of the interface IC: (i) the electrochemical sensor control and readout circuit, (ii) the Commands, and (iii) the Data transmission.

\section{A. Electrochemical Sensor Control and Readout}

The electrochemical control and readout block performs both $\mathrm{CV}$ and $\mathrm{CA}$ measurements on the selected bio-molecular sensor. The control and readout is achieved through a chain of analog and digital blocks. A fixed or triangular voltage waveform is generated internally by a direct digital synthesizer (DDS). The DDS includes the digital Refresh Unit and a 9-bit digital to analog converter (DAC). This voltage waveform is applied to the sensor selected by the multiplexer. The sensor current is converted to voltage through a transimpedance amplifier and later amplified by the Gain stage.

The output of the Gain stage goes through a sigma-delta modulator and a cascaded integrator-comb (CIC) filter to be digitized. A second order sigma-delta modulator is designed to work with an oversampling ratio of 256 and the signal bandwidth of $1 \mathrm{kHz}$ to achieve 13 bits dynamic range. The required clock and reference voltages are generated on-chip by the clock generator and the bandgap reference circuit, respectively. The 13 bit output of the CIC filter is given to the $T x / R x$ interface to prepare for transmission.

To reduce the power consumption and the area of the IC, the DDS is shared among different circuits to generate three different voltage profiles: (i) triangular waveform for CV; (ii) fixed voltage for $\mathrm{CA}$; (iii) triangular voltage for electrode conditioning. The sample and hold $(\mathrm{SH})$ circuits are used to enable DDS sharing when different voltage profiles are needed at the same time. More information about the design and simulation of the analog circuits in the readout chain can be found in [15].

\section{B. Commands}

The instruction set of the interface IC consists of three main commands, each having a length of two bytes: (i) Configuration; (ii) Execution; and (iii) Read. The Tx/Rx Interface unit samples the voltage on the Commands pin to extract the commands.

The instruction Configuration is used to define the measurement parameters. Two types of measurements can be performed by the biosensor array: CA and CV. Configuration [13:10] selects the measurement type and the parameter to set. The parameter is set to Configuration [8:0]. In case of $\mathrm{CV}$ measurement, four parameters can be set: the maximum voltage, the minim voltage, the scan rate, and the number of periods of the triangular waveform applied to the WE. In case of CA measurement, the applied voltage to the WE can be set. The circuits Conditioning 1 and Conditioning 2 are used 


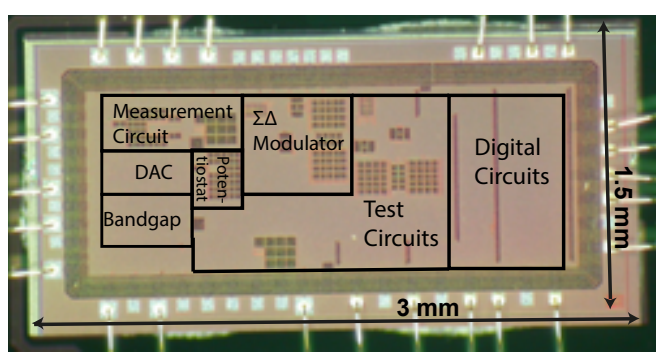

Fig. 3. The microphotograph of the interface IC.

to condition the electrodes when used for the first time and they can be configured in either $\mathrm{CA}$ or $\mathrm{CV}$ with configurable parameters. The connection of the five WEs and the RE with the measurement circuit and the conditioning circuits is also determined by the Configuration command. The command unit writes the parameters into the dedicated registers. The Refresh unit generates the digital data for the DAC as well as for the SH control signals.

The instruction Execution is used to begin/stop a CV, CA, or conditioning by applying/removing the appropriate voltage profile to the sensor. The stop command turns also off the sigma-delta modulator and the CIC filter. The instruction Read requests the readout IC to send back a certain number of measured data.

\section{Data Transmission}

If a Read command is received, the IC sends out the requested number of data. In case of $\mathrm{CA}$ or $\mathrm{CV}$ measurement, Data[12:0] contains the output of the sigma-delta ADC (i.e. the sensor current), while Data[15:13] of four consequent Data gives the 9-bit applied voltage (to the selected WE) preceded by a 3-bit starting pattern. An $8 \mathrm{~b} / 10 \mathrm{~b}$ channel encoding is used in the $T x / R x$ unit to convert the Data in order to achieve DCbalancing and avoid long sequences of the same logic value.

\section{Electrical Measurements}

The interface IC is implemented in $0.18 \mu \mathrm{m}$ CMOS technology. Its microphotograph is shown in Fig. 3. A FPGA board (Xilinx Virtex-5) is used to send the commands to the interface IC, and receive the data back from the IC. The Data bitstream is $8 \mathrm{~b} / 10 \mathrm{~b}$ decoded by the FPGA to extract the current and voltage values. Any fixed or triangular waveform with a slope larger than $2 \mathrm{~V} / \mathrm{S}$ is generated by commanding the IC to work in CA or CV modes, respectively. To generate the triangular waveform with a slope smaller than $2 \mathrm{~V} / \mathrm{S}$, the IC is commanded to work in the CA mode, and the slow triangular voltage is generated by sending a configuration command to the IC that changes the voltage of the WE, for every step in the voltage. The electrochemical readout circuit is characterized by configuring the IC for $\mathrm{CV}$ measurement. The proper Configuration commands are sent to the IC to generate and apply a triangular voltage waveform to WE1 with a maximum of $1.5 \mathrm{~V}$, minimum of $0.5 \mathrm{~V}$, and slope of $4.5 \mathrm{~V} / \mathrm{s}$. The circuit is electrically characterized by using a resistor (267 $\mathrm{k} \Omega$ ) instead of the sensor between WE1 and a fixed voltage

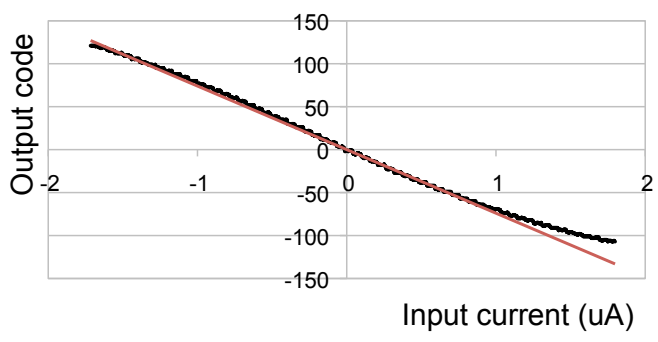

Fig. 4. Input-output characteristics of the current readout chain which includes the transimpedance amplifier, the gain stage, and the sigma-delta ADC.

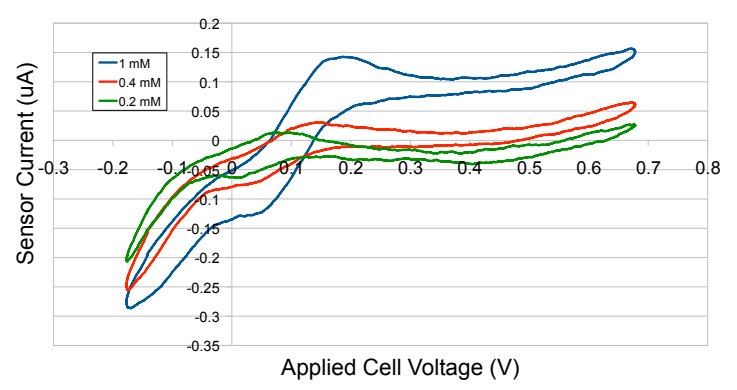

Fig. 5. Cyclic voltammetry of potassium ferricyanide at different concentrations with the IC commanded by the FPGA board.

of $900 \mathrm{mV}$. The input-output characteristics of the readout chain is shown in Fig. 4. The current readout chain includes the transimpedance amplifier, the gain stage, and the sigmadelta ADC. Measurements show that the 1-dB compression point in the current readout chain is at $\pm 1650 \mathrm{nA}$, while the readout resolution is 8 bits within this range. The measured power consumption of the IC is $0.92 \mathrm{~mW}$ from $1.8 \mathrm{~V}$ supply voltage.

\section{Electrochemical Measurements}

The interface IC is validated on the electrochemical sensors described in [16]. The same FPGA board is used with a different set of commands to address $\mathrm{CA}$ and $\mathrm{CV}$ measurements. Potassium ferricyanide $\left(\mathrm{K}_{4} \mathrm{Fe}(\mathrm{CN})_{6}\right)$ and hydrogen peroxide $\left(\mathrm{H}_{2} \mathrm{O}_{2}\right)$ are chosen as target molecules. The first compound is chosen because it has a well-defined response and highly reversible electrochemistry [3]. Therefore, it is a valid benchmark for cyclic voltammetry. The hydrogen peroxide, instead, is selected because it is an extremely significant molecule in the biomedical field. Many analytes can be detected by using oxidases. These enzymes usually promote a redox reaction, where the $\mathrm{H}_{2} \mathrm{O}_{2}$ is the main by-product [17]. Then, since hydrogen peroxide is an electrochemically active molecule, it can be directly detected through amperometric measurements.

\section{A. Validation of the cyclic voltammetry}

For each measurement, a drop of buffer saline solution with different concentration of potassium ferricyanide is placed on top of the WE, as well as the RE and the CE. A triangular voltage waveform with a maximum of $1.5 \mathrm{~V}$, minimum of 0.5 $\mathrm{V}$, and the slope of $10 \mathrm{mV} / \mathrm{sec}$ is applied to $\mathrm{WE} 1$ while 0.7 $\mathrm{V}$ is applied to RE. CV performed by the interface IC in the 


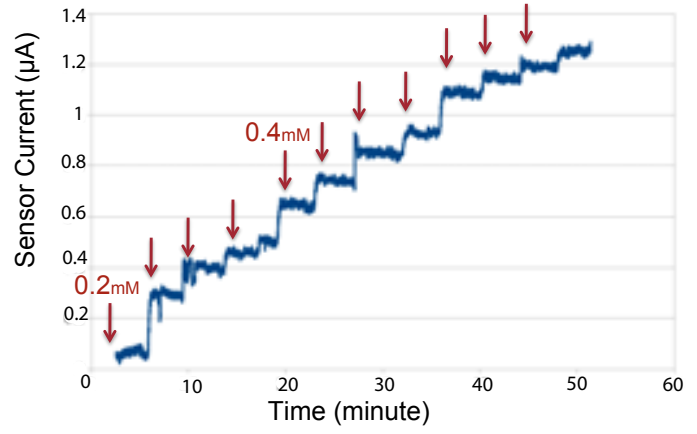

Fig. 6. Chronoamperometry of hydrogen peroxide using the proposed IC. Successively injections of $0.2 \mathrm{mM}$ and $0.4 \mathrm{mM}$ of $\mathrm{H}_{2} \mathrm{O}_{2}$ show that the IC is able to correctly readout the current.

TABLE I

MEASURED SPECIFICATIONS OF INTERFACE IC

\begin{tabular}{cc}
\hline Parameter & Value \\
\hline Current consumption of analog part & $462 \mu \mathrm{A}$ \\
Current consumption of digital part & $56 \mu \mathrm{A}$ \\
Minimum control voltage step & $3.5 \mathrm{mV}$ \\
Linear sensor current range & $\pm 1650 \mathrm{nA}$ \\
Current resolution & $13 \mathrm{nA}$ \\
\hline
\end{tabular}

presence of potassium ferricyanide at different concentrations is plotted in Fig. 5. The measured sensor current is calculated from the output code of the readout IC and the measured characteristics of the IC in Fig. 4. A low pass filter is used to reduce the noise. The places of the peaks agree well with literature [18].

\section{B. Validation of the chronoamperometry}

The CA of $\mathrm{H}_{2} \mathrm{O}_{2}$ at $+650 \mathrm{mV}$ is reported in Fig. 6. The sensor current is measured in time while the concentration of the $\mathrm{H}_{2} \mathrm{O}_{2}$ is increased in steps of $0.2 \mathrm{mM}$ or $0.4 \mathrm{mM}$. Since the CV and CA readout circuits are exactly the same, the IC output code in CA is converted to current using the same characteristics shown in Fig. 4. Table I summarizes the measurement results.

\section{CONCLUSION}

We presented a mixed signal interface IC for an electrochemical sensing data acquisition chain. The IC controls and reads out up to five biomolecular sensors. The IC supports both $\mathrm{CA}$ and $\mathrm{CV}$ measurements. Different voltage profiles are generated to control CV and CA by using a single fully on-chip waveform generator. The waveform generator is commanded by a standard interface for CA or CV with slope larger than 2 $\mathrm{V} / \mathrm{s}$ or by voltage steps for CV with slope smaller than $2 \mathrm{~V} / \mathrm{s}$. The IC reads out the electrochemical sensors and streams out the digitized measured data. The interface IC is implemented in $0.18 \mu \mathrm{m}$ CMOS technology and consumes $0.92 \mathrm{~mW}$ from $1.8 \mathrm{~V}$ supply voltage. The measured linear current readout range is $\pm 1650 \mathrm{nA}$ with 8-bit resolution. Electrochemical measurements including $\mathrm{CA}$ and $\mathrm{CV}$ measurement have been successfully performed on $\mathrm{H}_{2} \mathrm{O}_{2}$ and potassium ferricyanide, respectively. The encouraging results obtained from the IC make it ready to be integrated on the sensing platform.

\section{ACKNOWLEDGMENT}

The authors would like to thank Dr. Olivo and Dr. Cavallini for the help given for this project.

\section{REFERENCES}

[1] G. De Micheli, S. Ghoreishizadeh, C. Boero, F. Valgimigli, and S Carrara, An integrated platform for advanced diagnostics, in Design, Automation Test in Europe Conference Exhibition, pp. 16, 2011.

[2] B. B. Spear, M. Health-Chiozzi, and J. Huff, Clinical application of pharmacogenetics, Trends in Molecular Medicine, vol. 7, pp. 201-204, 2001.

[3] A. J. Bard and L. R. Faulkner, Electrochemical methods: fundamentals and applications, Wiley, New York, 1980.

[4] M.M. Ahmadi, G.A. Jullien, Current-mirror-based potentiostats for three-electrode amperometric electrochemical sensors, IEEE Transactions on Circuits and Systems I: Regular Papers, vol.56, no.7, pp.13391348, 2009.

[5] M. Haider, S. Islam, S. Mostafa, M. Zhang, and T. Oh, Low-power low voltage current readout circuit for inductively powered implant system, IEEE Transactions on Biomedical Circuits and Systems, vol. 4, no. 4, pp. $205213,2010$.

[6] L. Yu-Te, Y. Huanfen, A. Lingley, B. Parviz, B.P. Otis, A 3- $\mu W C M O S$ Glucose Sensor for Wireless Contact-Lens Tear Glucose Monitoring, IEEE Journal of Solid-State Circuits, vol. 47, no. 1, pp. 335-344, 2012.

[7] M. Stanacevic, K. Murari, A. Rege, G. Cauwenberghs, N. V. Thakor, VLSI Potentiostat Array With Oversampling Gain Modulation for WideRange Neurotransmitter Sensing, IEEE Transactions on Biomedical Circuits and Systems, vol.1, no.1, pp.63,72, 2007.

[8] A. Hassibi, T. H. Lee, A Programmable 0.18- um CMOS Electrochemical Sensor Microarray for Biomolecular Detection, IEEE Sensors Journal, vol. 6, no. 6, pp. 1380-1388, 2006.

[9] R.J. Reay, S.P. Kounaves, G.T.A. Kovacs, An integrated CMOS potentiostat for miniaturized electroanalytical instrumentation, IEEE international Solid-State Circuits Conference, pp. 162-163, 1994.

[10] M. Nazari and R. Genov, A fully differential CMOS potentiostat, IEEE International Symposium on Circuits and Systems, pp. 2177-2180, 2009.

[11] H. M. jafari, R. Genov, Chopper-stabilized bidirectional current acquisition circuits for electrochemical biomolecular detection, IEEE Transactions on Circuits and Systems I: Regular Papers, vol. 60, no. 5, pp. 1149-1157, 2013.

[12] P. Levine, P. Gong, R. Levicky, K. L. Shepard, Active CMOS sensor array for electrochemical biomolecular detection, IEEE Journal of SolidState Circuits, vol. 43, no. 8, pp. 1859-1871, 2008.

[13] L. Li, L. Xiaowen, W. A. Qureshi, A. J. Mason, CMOS Amperometric Instrumentation and Packaging for Biosensor Array Applications, IEEE Transactions on Biomedical Circuits and Systems, vol.5, no.5, pp. 439448, 2011.

[14] S. Ghoreishizadeh, C. Baj-Rossi, A. Cavallini, S. Carrara and G. De Micheli, An integrated control and readout circuit for implantable multitarget electrochemical biosensing, IEEE transactions on Biomedical Circuits and Systems, Accepted.

[15] S. Ghoreishizadeh, S. Carrara and G. De Micheli, A configurable IC to contol, readout, and Calibrate an array of biosensors, European conference in circuit theory and design (ECCTD), 2013.

[16] A. Cavallini, C. Baj-Rossi, S.S. Ghoreishizadeh, G. De Micheli, S. Carrara, Design, fabrication, and test of a sensor array for perspective biosensing in chronic pathologies, IEEE Biomedical Circuits and Systems Conference (BioCAS), pp. 124-127, 2012.

[17] I. Taurino, S. Carrara, M. Giorcelli, A. Tagliaferro, G. De Micheli, Comparing sensitivities of differently oriented multi-walled carbon nanotubes integrated on silicon wafer for electrochemical biosensors, Sensors and Actuators B: Chemical, pp. 327-333, 2011.

[18] G. Perenlei, et al. Voltammetric detection of potassium ferricyanide mediated by multi-walled carbon nanotube/titanium dioxide composite modified glassy carbon electrode, Int. J. Electrochem. Sci, pp. 520-531, 2011. 